DOI: 10.1515/awutm -2015-0001

思
Analele Universităţii de Vest, Timişoara

Seria Matematică - Informatică

LIII, 1, (2015), 3- 17

\title{
Factorizations in preduals associated to $\rho$-contractions
}

\author{
Bernard Chevreau and Aurelian Crăciunescu
}

\begin{abstract}
We establish directly factorization results for classes of $\rho$-contractions, corresponding to those obtained for certain classes of contractions. As an example of result which is not an immediate consequence of the fact that any $\rho$-contraction is similar to an ordinary contraction we give an "optimal" characterization of absolutely continuous $\rho$-contractions whose associated functional calculus has Property $\left(\mathbf{A}_{\aleph_{0}}\right)$. Along the way we show that a $\rho$-contraction is absolutely continuous if and only if it admits an absolutely continuous unitary $\rho$-dilation generalizing a wellknown result for usual contractions.
\end{abstract}

AMS Subject Classification (2000). Primary: 47A15, Secondary: 47A20, 47A60

Keywords. $\rho$-contraction, dilation, factorization, functional calculus.

\section{Introduction.}

It is well-known that dual algebra techniques have led to interesting results in terms of existence of invariant subspaces and structure information (predual factorization, reflexivity ...) particularly for certain classes of (Hilbert space) contractions (cf. for example [3], for an early - up to 1985 - survey, and $[2],[1],[5],[7],[4],[6]$ for later results). These results are easily seen to be "qualitatively" preserved by similarity. 
Among operators similar to contractions are the $\rho$-contractions that is those admitting a $\rho$-unitary dilation (see definition in next section) for some $\rho>0$. These operators (called also $\mathcal{C}_{\rho}$-contractions) were introduced by Sz.-Nagy and Foias who also established their similarity to contractions (see [9]). Therefore the classical results obtained for contractions via dual algebra theory have corresponding ones for $\rho$-contractions, e.g., any $\rho$-contraction whose spectrum contains the unit circle has nontrivial invariant subspaces, the functional calculus of any absolutely continuous $\rho$-contraction with essential spectrum dominating has Property $\left(\mathbf{A}_{\aleph_{0}}\right)$; indeed this follows respectively from the results in [2], [3] Chap. V. In fact, looser hypothesis related to the so-called approximating sets (see definitions below) will also lead to the same last factorization result. However the similarity to contractions does not preserve certain constants appearing in these processes. In particular it does not seem possible to deduce the optimal characterizations of the analogues of the classes $\mathbf{A}_{\aleph_{0}}$ and $\mathbf{A}_{1, \aleph_{0}}$ from this similarity result.

These observations motivate our attempt to study directly (that is without using the above similarity result) these factorizations in the predual of dual algebras generated by $C_{\rho}$-contractions. As a first step in this program, in this note we do obtain the "best" characterization of the class of absolutely continuous $\rho$-contractions whose functional calculus has Property $\left(\mathbf{A}_{\aleph_{0}}\right.$ ) (analogue of the class $\mathbf{A}_{\aleph_{0}}$ ) in terms of approximating sets (cf. our main result, Theorem 3.4. below).

The paper is organized as follows: in Section 2 after recalling basic definitions and terminology we characterize absolutely continuous $\rho$-contractions, give a property sort of saying what is preserved for general $\rho$-contractions of the existence for usual contractions (i.e. 1-contractions) of a coisometric extension. This is exploited in the last section to connect spectral properties with membership in approximating sets; this is a key ingredient in the proof of our main result.

\section{Preliminaries}

Let $\mathcal{H}$ be a complex, infinite dimensional, separable Hilbert space and let $L(\mathcal{H})$ denote the Banach algebra of (bounded linear) operators on $\mathcal{H}$. As usual, for $T \in L(\mathcal{H}), \sigma_{l e}(T), \sigma_{r e}(T)$ and $\sigma_{e}(T)$ denote respectively the left essential, right essential and essential spectrum of $T$.

We denote by $A(\mathbb{D})$ the disc algebra, that is, the algebra of complex functions continuous on the closed unit disc and analytic in $\mathbb{D}$ and by $H^{\infty}=$ $H^{\infty}(\mathbb{D})$ the algebra of bounded analytic functions in the open unit disc 
(equipped with the sup norm $\left(\|h\|_{\infty}=\sup _{z \in \mathbb{D}}|h(z)|\right)$. Alternatively $H^{\infty}=H^{\infty}(\mathbb{T})$ that is, as well-known, it can be viewed as the subalgebra of $L^{\infty}(\mathbb{T})$ consisting of the (classes of Lebesgue-measurable) functions whose Fourier coefficients of (strictly) negative index vanish. As such it is a weak*closed subalgebra of $L^{\infty}(\mathbb{T})$ whose predual is the quotient space $Q=L^{1} / H_{0}^{1}$. It is also well-known that $H^{\infty}$ is the weak*-closure of $A(\mathbb{D})$ in $L^{\infty}(\mathbb{T})$. More precisely $A(\mathbb{D})$ is strongly sequentially pointwise boundedly dense in $H^{\infty}$ that is, for any $f$ in $H^{\infty}$ there exist a sequence $\left(g_{n}\right)$ in $A(\mathbb{D})$ converging pointwise $($ in $\mathbb{D})$ to $f$ and satisfying $\left\|g_{n}\right\|_{\infty} \leq\|f\|_{\infty}, n \in \mathbb{N}$. Recall that for $\lambda$ in $\mathbb{D}$ the linear functional (point evaluation at $\lambda) f \rightarrow f(\lambda)$ is weak ${ }^{*}$ continuous on $H^{\infty}$ and thus can be identified with an element $E_{\lambda}$ in $Q$. Hence the kernel $H_{0}^{\infty}\left(=\alpha H^{\infty}\right)$ (with $\alpha$ the position function) of the evalutation at 0 is a weak ${ }^{*}$-closed subspace of $H^{\infty}$ and we have the (weak* as well as norm) topological direct sum decomposition $H^{\infty}=\mathbb{C} 1 \oplus H_{0}^{\infty}$.

For $\rho>0$, an operator $T \in L(\mathcal{H})$ is said to be of class $C_{\rho}$ (or a $\rho$ contraction), if there is a Hilbert space $\mathcal{K} \supset \mathcal{H}$ and a unitary operator $U \in L(\mathcal{K})$ such that

$$
T^{n} x=\rho P_{\mathcal{H}} U^{n} x, n \in \mathbb{N}, x \in \mathcal{H},
$$

where $P_{\mathcal{H}}$ is the orthogonal projection of $\mathcal{K}$ on $\mathcal{H}$. The unitary operator $U$ is called a $\rho$-unitary dilation of $T$ and, provided it is minimal in the sense that $\mathcal{K}=\vee_{n \in \mathbb{Z}} U^{n} \mathcal{H}$, it is uniquely determined up to unitary equivalence. The adjoint of a $\rho$-contraction is also a $\rho$-contraction and the spectral radius $r(T)$ of a $\rho$-contraction satisfies $r(T) \leq 1$ and therefore its spectrum is included in the closure of the unit disk, $\mathbb{D} \subset \mathbb{C}$. We recall that a $\rho$-contraction is polynomially bounded, that is, $T$ being a $\rho$-contraction, there exists a constant $M$ such that for any polynomial $p$ we have

$$
\|p(T)\| \leq M\|p\|_{\infty}
$$

where

$$
\|p\|_{\infty}=\sup _{z \in \mathbb{D}}|p(z)|=\sup _{z \in \mathbb{T}}|p(z)| .
$$

In fact (cf. [10]), we can take $M=\rho+|\rho-1|$.

As well-known, an operator $T \in \mathcal{L}(\mathcal{H})$ is polynomially bounded if and only if it admits a (unital, norm continuous) representation $\Phi_{T}$ of the disc algebra $A(\mathcal{D})$ into $\mathcal{L}(\mathcal{H})$, extending the polynomial calculus associated to $T$ - Recall that a polynomially bounded operator $T$ is said to be absolutely continuous if the representation $\Phi_{T}$ admits a weak*-continuous extension to 
$H^{\infty}$ (which we still denote $\Phi_{T}$ and write as usual $h \rightarrow h(T)$ ). Here the weak*topology of $\mathcal{L}(\mathcal{H})$ is that coming from the classical duality $\left.\mathcal{L}(\mathcal{H})=\mathcal{C}_{1}(\mathcal{H})\right)^{*}$ where $\mathcal{C}_{1}(\mathcal{H})$ is the Banach space of trace class operators.

The following proposition gives convenient characterizations of absolute continuity for polynomially bounded operators.

Proposition 2.1. Let $T \in \mathcal{L}(\mathcal{H})$ be a polynomially bounded operator and $\Phi$ the associated representation of $A(\mathbb{D})$ into $\mathcal{L}(\mathcal{H})$; then the following assertions are equivalent:

a) $T$ is absolutely continuous,

b) Whenever a sequence $\left(p_{n}\right)_{n}$ in $A(\mathbb{D})$ converges pointwise boundedly to 0 the sequence $\left(p_{n}(T)\right)_{n}$ converges weak* to 0 in $\mathcal{L}(\mathcal{H})$,

c) For any $x, y$ in $\mathcal{H}$ the linear functional $p \rightarrow(p(T) x, y)$ is weak*continuous $\left(A(\mathbb{D})\right.$ being equipped with the relative weak ${ }^{*}$-topology).

Proof. In view of the characterization of sequential weak*-convergence in $H^{\infty}(\mathbb{D})$ the implication $\left.\left.a\right) \Rightarrow b\right)$ is immediate.

Since, in $\mathcal{L}(\mathcal{H})$, weak*-convergence and weak-operator convergence coincide for bounded sequences we have the equivalence $b) \Leftrightarrow c$ ).

The remaining implication " $b) \Rightarrow c$ )" can be proved along the lines of [8], p.152 (cf. also [6] or [11]). For the sake of completeness we sketch below a somewhat more elementary argument.

Recall that whenever the Banach space $X$ is separable there exists a translation-invariant metric $d$ on $X^{*}$ such that on any closed ball in $X^{*}$ the weak*-topology coincides with that induced by the metric $d$ (indeed take a countable dense set $\left\{x_{n} ; n \geq 1\right\}$ in the unit ball of $X$, then $d(f, g):=$ $\Sigma_{n} 2^{-n}\left|<x_{n}, g-f>\right|$ will do). We choose then $d$ and $d^{\prime}$ two such metrics on respectively $H^{\infty}=(\mathcal{Q})^{*}$ and $\mathcal{L}(\mathcal{H})=\left(\mathfrak{C}_{1}(\mathcal{H})\right)^{*}$. Let $B$ (resp. $B^{\prime}$ ) the closed unit balls in $H^{\infty}$ (resp. $\mathcal{L}(\mathcal{H})$ ); given $r>0$, the continuity of $\Phi$ at 0 together with the invariance by translation of the metrics $d_{1}, d_{2}$ yields easily the uniform continuity of $\Phi_{r}:=\Phi_{\mid r B \cap A(\mathbb{D})}$. The map $\Phi_{r}$, uniformly continuous on the dense subset $r B \cap A(\mathbb{D})$ and valued in the complete metric space $r^{\prime} B^{\prime}$ (with $r^{\prime}=r\|\Phi\|$ ), has a unique (uniformly) continuous extension $\tilde{\Phi}_{r}$ to $r B$. Since $\tilde{\Phi}_{r^{\prime}}$ is clearly an extension of $\tilde{\Phi}_{r}$ whenever $r^{\prime} \geq r$, setting $\tilde{\Phi}(f)=\tilde{\Phi}_{r}(f)$ for $f$ in $H^{\infty}$ and $r \geq\|f\|$ we obtain a well-defined extension $\tilde{\Phi}$ of $\Phi$ to all of $H^{\infty}$ satisfying $\|\tilde{\Phi}(f)\| \leq\|\Phi\|\|f\|$. The linearity of $\tilde{\Phi}$ is deduced from that of $\Phi$ via a routine argument (for scalars $\lambda, \mu$ and $f, g$ in $H^{\infty}$ choose $r \geq \max \{\|f\|,\|g\|,|\lambda|\|f\|+|\mu|\|g\|\}$ and sequences $\left(u_{n}\right)_{n},\left(v_{n}\right)_{n}$ in $A(\mathbb{D})$ converging pointwise to, respectively, $f, g$ and satisfying 
$\left\|u_{n}\right\| \leq\|f\|,\left\|v_{n}\right\| \leq\|g\|$ for all $n$ etc..). Thus $\tilde{\Phi}$ is a bounded linear map from $H^{\infty}$ into $\mathcal{L}(\mathcal{H})$ which, by construction, is sequentially weak ${ }^{*}$-continuous, hence weak*-continuous by a standard argument. The multiplicativity of $\tilde{\Phi}$ is proved by the usual 2 steps argument (starting with $\Phi\left(u_{n} v_{n}\right)=\Phi\left(u_{n}\right) \Phi\left(v_{n}\right)$ for $u_{n}, v_{n}$ in $A(\mathbb{D})$ we obtain $\tilde{\Phi}\left(f v_{n}\right)=\tilde{\Phi}(f) \Phi\left(v_{n}\right)$ for $f$ in $H^{\infty}, v_{n}$ in $A(\mathbb{D})$ and next $\tilde{\Phi}(f g)=\tilde{\Phi}(f) \tilde{\Phi}(g)$ for $f, g$ in $\left.H^{\infty}\right)$.

From now on, when $T$ is an absolutely continuous polynomially bounded operator with $\Phi_{T}$ the associated weak ${ }^{*}$-continuous representation, for $x, y$ in $\mathcal{H}$ we denote by $x \square_{T} y$ (just $x \square y$ if there is no ambiguity on the operator under consideration) the element of $\mathcal{Q}$ defined by

$$
<x \square_{T} y, f>=(f(T) x, y),
$$

$f \in H^{\infty}$.

Before discussing absolute continuity for $\rho$-contractions we gather some easy (and well-known) facts about this sesquilinear map $\square_{T}$. Recall that a semi-invariant subspace for an operator $T$ is the orthogonal difference of two nested invariant subspaces. If $\mathcal{J}=\mathcal{M} \ominus \mathcal{N}(\mathcal{M}, \mathcal{N} \in \operatorname{Lat} T, \mathcal{N} \subset \mathcal{M})$ is such a subspace we write $T_{\mathfrak{J}}$ for the compression of $T$ to $\mathcal{J}$, that is, the operator defined on $\mathcal{J}$ by $T_{\mathfrak{J}}(x)=P_{\mathfrak{g}} T x, x \in \mathcal{J}$.

Proposition 2.2. Let $T \in \mathcal{L}(\mathcal{H})$ be an absolutely continuous polynomially bounded operator.

a) For any $x, y \in \mathcal{H}$ and $g \in H^{\infty}$ we have $g(T) x \square y=x \square(g(T))^{*} y$.

b) Let $\mathcal{M} \in \operatorname{LatT}, T_{0}=T_{\mid \mathcal{M}}, P_{\mathcal{M}}$ the orthogonal projection on $\mathcal{M}, x \in \mathcal{M}$ and $y \in \mathcal{H}$, then

$$
x \square_{T} y=x \square_{T} P_{\mathcal{M}} y=x \square_{T_{0}}\left(P_{\mathcal{M}} y\right) .
$$

Similarly, let $\mathcal{F} \in$ LatT $^{*}, T_{1}$ the compression of $T$ to $\mathcal{F}$ (in this case $\left.\left(T_{1}\right)^{*}=\left(T^{*}\right)_{\mid \mathcal{F}}\right), x \in \mathcal{H}$ and $y \in \mathcal{F}$; then $x \square_{T} y=\left(P_{\mathcal{F}} x\right) \square_{T} y=$ $\left(P_{\mathcal{F}} x\right) \square_{T_{1}} y$.

c) If $\mathcal{J}$ is a semi-invariant subspace for $T$, then for $x, y \in \mathcal{J}, x \square_{T_{\mathfrak{J}}} y=$ $x \square_{T} y$.

d) Let $\left(x_{i}\right)_{i \in J}$ be a sequence of vectors $(J \subset \mathbb{N}$, finite or not); the map $y \rightarrow\left(x_{i} \square y\right)_{i \in J}$ from $\mathcal{H}$ into $J^{\mathcal{Q}}$ (the space of $\mathcal{Q}$ valued sequences indexed by $J$ ) has kernel $\mathcal{M}^{\perp}$ where $\mathcal{M}$ is the invariant subspace for $T$ generated by the $x_{i}^{\prime} s$. 
The following proposition generalizes to $\rho$-contractions the corresponding (well-known) result for usual contractions.

Proposition 2.3. Let $T \in \mathcal{L}(\mathcal{H})$ be a $\rho$-contraction; then $T$ is absolutely continuous iff it admits an absolutely continuous unitary $\rho$-dilation.

Proof. Let $U \in \mathcal{L}(\mathcal{K})$ a unitary $\rho$-dilation of $T \in \mathcal{L}(\mathcal{H})$; for any polynomial $p$ and any $x \in \mathcal{H}$ we have

$$
p(T) x=p(0) x+\rho P_{\mathcal{H}} q(U) x,
$$

where $q=p-p(0)$. This equality extends by continuity to any $p \in A(\mathbb{D})$ and the implication " $U$ absolutely continuous $\Rightarrow T$ absolutely continuous" follows easily.

Suppose, to prove the converse, that the $\rho$-contraction $T$ (with $\rho$-unitary dilation $U$ ) is absolutely continuous; we may and do assume that $U$ is minimal, that is, $\mathcal{K}=\bigvee_{j \in \mathbb{Z}} U^{j} \mathcal{H}$. Let then $\left(h_{n}\right)_{n}$ a sequence in $A(\mathbb{D})$ converging weak* to 0 and let

$$
\mathcal{G}:=\left\{(u, v) \in \mathcal{K} \times \mathcal{K} \mid\left(h_{n}(U) u, v\right) \rightarrow 0\right\} .
$$

To avoid confusion between ordered pairs and inner products, down to the end of this proof, we write $\langle x, y\rangle$ for the inner product.

We wish to show that $\mathcal{G}=\mathcal{K} \times \mathcal{K}$. Obviously $\mathcal{G}$ is a closed linear subspace (just use the norm-boundedness of $\left.\left(h_{n}(U)\right)_{n}\right)$; thus, by the minimality hypothesis, it suffices to show that any given element $(u, v)$ of the form $(u, v)=\left(U^{j} x, U^{k} y\right)(j, k \in \mathbb{Z}, x, y \in \mathcal{H})$ is in $\mathcal{G}$.

a) for $j>k$

$$
<h_{n}(U) u, v>=<h_{n}(U) U^{j-k} x, y>=\frac{1}{\rho}<h_{n}(T) T^{j-k} x, y>
$$

that is,

$$
<h_{n}(U) u, v>=\frac{1}{\rho}<T^{j-k} x \square y, h_{n}>
$$

and hence $\left(U^{j} x, U^{k} y\right) \in \mathcal{G}$ in this case;

b) for $j \leq k$ we write

$$
h_{n}=p_{n}+\alpha^{k-j+1} g_{n}
$$

where $p_{n}:=\Sigma_{i \leq k-j} \frac{1}{i !} h_{n}^{(i)}(0) \alpha^{i}$ is the Taylor polynomial of degree $k-j$ of $h_{n}$ at the origin; thus

$$
<h_{n}(U) u, v>=<p_{n}(U) U^{j} x, U^{k} y>+<U^{k-j+1} g_{n}(U) U^{j} x, U^{k} y>
$$


the Taylor coefficients are weak*-continuous, therefore the sequence $\left(p_{n}\right)$ converges to 0 in norm which yields first that $\left(g_{n}\right)$ converges weak ${ }^{*}$ to 0 and second that the first term on the right hand side of the above equality tends to 0 ; as to second term we have

$$
<U g_{n}(U) x, y>=\frac{1}{\rho}<T g_{n}(T) x, y>=\frac{1}{\rho}<T x \square y, g_{n}>\rightarrow 0 .
$$

(We remark that Case $b$ ) could be deduced from $a$ ) using the fact that $U^{*}$ is a unitary $\rho$-dilation of $T^{*}$ and treating apart -via the decomposition $h_{n}=h_{n}(0)+\alpha g_{n^{-}}$the special case $j=k$.)

We now review the factorization properties.

Definition 2.4. Given cardinal numbers, $0<m, n \leq \aleph_{0}$, we say that the (absolutely continuous) representation $\Phi_{T}$ has Property $\left(\mathbb{A}_{m, n}\right)$ if given an $m \times n$ matrix with entries in $Q$,

$$
\begin{aligned}
\left(L_{i, j}\right) & 0 \leq i<m \\
& 0 \leq j<n
\end{aligned}
$$

there exist vectors $\left(x_{i}\right)_{0 \leq i<m}$ and $\left(y_{j}\right)_{0 \leq j<n}$ such that

$$
L_{i, j}=x_{i} \square_{T} y_{j}, 0 \leq i<m, 0 \leq j<m .
$$

We write $\left(\mathbb{A}_{n}\right)$ for $\left(\mathbb{A}_{n, n}\right)$.

In this note we will be mostly interested in the strongest of these properties, namely Property $\left(\mathbb{A}_{\aleph_{0}}\right)$. Recall that one essential approach to obtain this property is via the so-called approximation set $\mathfrak{X}_{\mathfrak{o}}\left(=\mathfrak{X}_{\mathfrak{o}}(T)\right)$ defined as the set of those elements $L \in \mathcal{Q}$ for which there exist sequences $\left(x_{n}\right)_{n}$ and $\left(y_{n}\right)_{n}$ in the closed unity ball of $\mathcal{H}$ such that

$$
\lim _{n \rightarrow \infty}\left\|L-x_{n} \square_{T} y_{n}\right\|=0,
$$

and

$$
\lim _{n \rightarrow \infty}\left(\left\|w \square_{T} y_{n}\right\|+\left\|x_{n} \square_{T} w\right\|\right)=0 .
$$

The importance of this set lies in the following proposition (cf. [5]).

Proposition 2.5. The set $\mathfrak{X}_{\mathrm{o}}$ is absolutely convex and closed. If it contains a ball of (strictly) positive radius then $\Phi_{T}$ does have Property ( $\mathbf{A}_{\aleph_{0}}$ ). 
We conclude this preliminary section with a property better expressed in the setting of coisometric $\rho$-dilation. Given $T \in L(\mathcal{H})$ a $\rho$-contraction, we consider a unitary $\rho$-dilation $U \in L(\mathcal{K})$ of $T^{*}$. Set $\mathcal{K}^{+}=\bigvee_{n \geq 0} U^{n} \mathcal{H}$, $U^{+}=U_{\left.\right|_{\mathcal{K}} ^{+}}$, and $B=\left(U^{+}\right)^{*}$. The $B$ operator is a coisometry called the minimal coisometrical $\rho$-dilation of $T$. In accordance with Wold's Theorem we will have $\mathcal{K}^{+}=\mathcal{S} \oplus \mathcal{R}$, with $\mathcal{S}, \mathcal{R} \in \operatorname{Lat} B$ and $B=S^{*} \oplus R$, where $S$ is a shift operator on $\mathcal{S}$, and $R$ is unitary on $\mathcal{R}$. Moreover

$$
\mathcal{S}=\bigvee_{n \geq 1} \operatorname{Ker} B^{n}=\bigvee_{\mu \in \mathbb{D}} \operatorname{Ker}(B-\mu)
$$

The first part of the following proposition, besides its usefulness later on, presents some independant interest in the sense that it shows what is preserved in general of the special $\rho=1$ case (where $B$ is an extension of $T$ ). The second part is just an easy but useful observation with a convenient reformulation based on the decomposition

$$
H^{\infty}=\mathbb{C} 1 \oplus H_{0}^{\infty}
$$

and the notations $\widehat{L}=L_{\mid H_{0}^{\infty}}, x \oslash_{T} y:=\widehat{x \square_{T} y}$.

Proposition 2.6. In the above context a), for $x \in \mathcal{H}$ and $n \in \mathbb{N}$ :

a) $B x-\operatorname{Tx} \in \operatorname{Ker} B$

b) For $x, y \in \mathcal{H}$

$$
T x \square_{T} y=\rho B x \square_{B} y,
$$

and consequently, $T x \oslash_{T} y=\rho B x \oslash_{B} y$.

c) Given an arbitrary sequence $\left(L_{n}\right)_{n}$ in $Q$ the following two assertions are equivalent:

(i) $L_{n} \rightarrow 0$

(ii) $\widehat{L_{n}} \rightarrow 0$ and $<1, L_{n}>\rightarrow 0$.

Proof. For $x, y \in \mathcal{H}$ and $n \in \mathbb{N}$ we will have

$$
\begin{aligned}
<B(B x-T x), B^{* n} y> & =<x, U^{n+2} y>-<T x, U\left(U^{n} y\right)>= \\
& =\frac{1}{\rho}<x,\left(T^{*}\right)^{n+2} y>-\frac{1}{\rho}<T x,\left(T^{*}\right)^{n+1} y>=0
\end{aligned}
$$


Therefore,

$$
B x-T x \in \operatorname{Ker} B
$$

This proves "a)".

We have also, for $h$ in $H^{\infty}$

$$
<T x \square_{T}, y, h>=(h(T) T x, y)=\rho(h(B) x, y)=<\rho B x \square_{B} y, h>
$$

that is "b)".

Finally "c)" results from the obvious fact that $Q$ is isomorphic to the direct sum $\mathbb{C} \oplus \mathcal{Q}_{0}$ where $\mathcal{Q}_{0}$ is the predual of $\alpha H^{\infty}$.

\section{The main result}

We begin with a lemma which establishes an essential connection between spectral properties and approximation set. We follow the usual notation for weak convergence of the sequence $\left(x_{n}\right)_{n}$ toward $x: x_{n} \rightarrow 0$.

LEMMA 3.1. Let $T \in L(\mathcal{H})$ be an absolutely continuous $\rho$-contraction and let $\lambda \in \sigma_{l e}(T) \cap \mathbb{D}$, then $E_{\lambda} \in \mathfrak{X}_{0}\left(=\mathfrak{X}_{0}(T)\right)$ via any sequences $\left(x_{n}\right)_{n},\left(x_{n}\right)_{n}$ such that $x_{n} \rightarrow 0,\left\|x_{n}\right\| \rightarrow 1$ and $\left\|(T-\lambda) x_{n}\right\| \rightarrow 0$. The same result holds if $\lambda \in \sigma_{r e}(T)$ and the sequence $\left(x_{n}\right)_{n}$ satisfies $x_{n} \rightarrow 0,\left\|x_{n}\right\| \rightarrow 1$ and $\left\|(T-\lambda)^{*} x_{n}\right\| \rightarrow 0$.

Proof. It is straightforward to check that, for any $x, y \in \mathcal{H}, \lambda \in \mathbb{D}$, we have $\left\|x \square_{T^{*}} y\right\|=\left\|y \square_{T} x\right\|$ and $\left\|E_{\lambda}-x \square_{T} y\right\|=\left\|E_{\bar{\lambda}}-y \square_{T^{*}} x\right\|$. From these equalities combined with the fact that $\lambda \in \sigma_{r e}(T)$ if and only if $\bar{\lambda} \in \sigma_{l e}\left(T^{*}\right)$ we see that if $E_{\bar{\lambda}}$ belongs to $\mathfrak{X}_{0}\left(T^{*}\right)$ via sequences $\left(x_{n}\right),\left(y_{n}\right)$ then $E_{\lambda}$ belongs to $\mathfrak{X}_{0}(T)$ via the sequences $\left(y_{n}\right),\left(x_{n}\right)$. It follows from these considerations that we just need to treat the case $\lambda \in \sigma_{l e}(T)$. (Note that, by the classical geometric characterization of the left essential spectrum, among the sequences $\left(x_{n}\right)_{n}$ satisfying the above conditions, there are orthonormal ones.) To show that $E_{\lambda}$ belongs to the approximation set $\mathfrak{X}_{0}$ via the sequences $\left(x_{n}\right),\left(x_{n}\right)$ we have three assertions to prove:

(i) (the approximation of $E_{\lambda}$ by the $\left.x_{n} \square x_{n}\right) \lim _{n \rightarrow \infty} a_{n}=0$ where $a_{n}:=$ $\left\|x_{n} \square x_{n}-E_{\lambda}\right\| ;$

(ii) (the vanishing condition on the right) given $w \in \mathcal{H}, \lim _{n \rightarrow \infty} b_{n}=0$ where $b_{n}:=\left\|x_{n} \square w\right\|$; 
(iii) (the vanishing condition on the left) given $w \in \mathcal{H}, \lim _{n \rightarrow \infty} c_{n}=0$ where $c_{n}:=\left\|w \square x_{n}\right\|$.

Among those three, the first two hold (with of course the same spectral hypothesis) for any absolutely continuous polynomially bounded operator. This is rather well known and we give the proof only for the sake of completeness.

Proof of (i): By the Hahn-Banach theorem, for each $n$, there exists $h_{n}$ of norm 1 in $H^{\infty}$ such that

$$
a_{n}=<x_{n} \square x_{n}-E_{\lambda}, h_{n}>=\left(h_{n}(T) x_{n}, x_{n}\right)-h_{n}(\lambda) .
$$

Writing

$$
h_{n}=h_{n}(\lambda)+(\alpha-\lambda) g_{n}
$$

with $g_{n}$ in $H^{\infty}$ and (via the maximum modulus principle) $\left\|g_{n}\right\| \leq \frac{2}{1-|\lambda|}$ we obtain (using also the fact that $\left\|x_{n}\right\|=1$ )

$$
a_{n}=\left(g_{n}(T)(T-\lambda) x_{n}, x_{n}\right),
$$

thus

$$
a_{n}\left(\leq 2(1-|\lambda|)^{-1}||(T-\lambda) x_{n}||\right) \rightarrow 0 .
$$

The proof of (ii) is similar: there exists a sequence $\left(h_{n}\right)$ of functions of norm 1 in $H^{\infty}$ such that (applying the same decomposition of $h_{n}$ as in (i))

$b_{n}=<x_{n} \square w, h_{n}>=\left(h_{n}(T) x_{n}, w\right)=h_{n}(\lambda)\left(x_{n}, w\right)+\left(g_{n}(T)(T-\lambda) x_{n}, w\right)$, thus, using the fact that $\left(x_{n}\right)$ tends weakly to 0 )

$$
b_{n}\left(\leq\left|\left(x_{n}, w\right)\right|+2(1-|\lambda|)^{-1}||(T-\lambda) x_{n}||\|w\|\right) \rightarrow 0 .
$$

As to Assertion (iii), since

$$
<w \square_{T} x_{n}, 1>\left(=\left(w, x_{n}\right)\right) \rightarrow 0
$$

in view of Prop.2.5. we just need to show that $w \oslash_{T} x_{n} \rightarrow 0$ or, equivalently, $w \oslash_{B} x_{n} \rightarrow 0$. To show this last convergence it obviously suffices to prove that $w \square_{B} x_{n} \rightarrow 0$.

But with the notations introduced about the Wold decomposition of $B$ it is easy to check that 


$$
w \square_{B} x_{n}=Q w \square_{S^{*}} Q x_{n}+A w \square_{R} A x_{n},
$$

(recall that $Q, A$ are orthogonal projections on the subspaces $\mathcal{S}$, respectively $\mathcal{R}$ of this Wold decomposition).

Again here it is well-known that, since $\left(Q x_{n}\right)$ tends weakly to 0 , the first term in the right hand side of the above equality will tend to 0.

(Sketch of a quick proof - with the "boxes" here referring exclusively to the operator $S^{*}-$ : The set

$$
\mathcal{V}:=\left\{z \in \mathcal{S} ; z \square Q x_{n} \rightarrow 0\right\}
$$

is a closed subspace of $\mathcal{S}$; for $\mu \in \mathbb{D}$ and $\left(S^{*}-\mu\right) z=0$ we have, for some $h_{n}$ of norm 1,

$$
\left\|z \square Q x_{n}\right\|=\left(h_{n}\left(S^{*}\right) z, Q x_{n}\right)=h_{n}(\lambda)\left(z, Q x_{n}\right) \rightarrow 0 .
$$

This shows that $\mathcal{V} \supset \operatorname{Span}_{\mu \in \mathbb{D}} \operatorname{ker}\left(S^{*}-\mu\right)$, that is $\mathcal{V}=\mathcal{S}$.)

We finally use Part a) of Proposition 2.2 to show that, like in the case of ordinary contractions, we have $A x_{n} \rightarrow 0$. This will finish the proof of the fact that $w \square_{B} x_{n} \rightarrow 0$, hence, that of (iii) and complete the proof of the lemma.

Indeed from $B x_{n}-T x_{n} \in \operatorname{Ker} B \subset \mathcal{S}$ it results that $A B x_{n}=A T x_{n}$. But $A B x_{n}=R A x_{n}$ and then

$$
(1-|\lambda|)\left\|A x_{n}\right\| \leq\left\|(R-\lambda) A x_{n}\right\|=\left\|A(B-\lambda) x_{n}\right\|=\left\|A(T-\lambda) x_{n}\right\| \leq\left\|(T-\lambda) x_{n}\right\|,
$$

yielding the desired conclusion.

A first consequence of this fundamental lemma is the ("direct") extension to absolutely continuous $\rho$-contractions of a classical sufficient spectral condition for the associated representation $\Phi_{T}$ to enjoy the $\left(\mathbb{A}_{\aleph_{0}}\right)$ property with the extra bonus (not immediately available via similarity to a contraction) that the approximation set is "as big as it can be". We recall that a subset $\Lambda \subset \mathbb{D}$ is said to be dominating if for any $h$ in $H^{\infty}$ we have $\|h\|_{\infty}=\sup _{\lambda \in \Lambda}|h(\lambda)|$. A straightforward application of the Hahn-Banach Theorem shows that this is equivalent to the fact the absolutely closed convex hull of $\left\{E_{\lambda} ; \lambda \in \Lambda\right\}$ is the closed unit ball $\mathcal{Q}_{1}$ of $\mathcal{Q}$. Since the approximation set $\mathfrak{X}_{0}$ is absolutely convex and closed we obtain the following result.

THEOREM 3.2. Let $T$ be an absolutely continuous $\rho$-contraction such that $\sigma_{e}(T) \cap \mathbb{D}$ is dominant in $\mathbb{D}$ then $\mathfrak{X}_{0}(T)=\mathcal{Q}_{1}$ and therefore $\Phi_{T}$ has the property $\left(\mathbb{A}_{\aleph_{0}}\right)$. 
Our main goal is to obtain an optimal converse of this result. To that end we first develop some more or less known consequences of the property $\left(\mathbb{A}_{\aleph_{0}}\right)$.

Proposition 3.3. Let $T$ be an absolutely continuous polynomially bounded operator such that the associated representation $\Phi_{T}$ has property $\left(\mathbb{A}_{\aleph_{0}}\right)$ and let $\lambda \in \mathbb{D}$; then there exists an infinite dimensional semi-invariant subspace I for $T$ such that $T_{\mathfrak{J}}$, the compression of $T$ to $\mathcal{J}$, is $\lambda I_{\mathfrak{J}}$.

Moreover if, in addition, $T$ is a $\rho$-contraction then $E_{\lambda} \in \mathfrak{X}_{0}(T)$ via sequences $\left(e_{n}\right)_{n},\left(e_{n}\right)_{n}$ where $\left(e_{n}\right)_{n}$ is any sequence of unit vectors in $\mathcal{J}$ converging weakly to 0 and consequently $\mathfrak{X}_{0}(T)=Q_{1}$.

Proof. We apply the definition of Property $\left(\mathbb{A}_{\aleph_{0}}\right)$ to the matrix $\left(\delta_{i, j} E_{\lambda}\right)_{i, j \in \mathbb{N}}$ and get sequences of vectors $\left(x_{i}\right),\left(y_{i}\right)$ such that

$$
\delta_{i, j} E_{\lambda}=x_{i} \square y_{j}, i, j \in \mathbb{N} .
$$

Observe that both sequences of vectors $\left(x_{i}\right)_{i}$ and $\left(y_{j}\right)_{j}$ are (algebraically) linearly independant (indeed, if some $x_{k}$ is a finite linear combination of the other $x_{i}$ 's, say $x_{k}=\Sigma_{i \neq k} \beta_{i} x_{i}$, we have an immediate contradiction upon expanding $x_{k} \square y_{k}$, namely $E_{\lambda}=0$; similarly for the $y_{j}$ 's).

Using Prop. 2.2. we note first that we may (and do) assume that the $y_{j}^{\prime} s$ belong to the invariant subspace $\mathcal{M}$ for $T$ generated by the $x_{i}^{\prime} s$, next that

$$
0=(T-\lambda) x_{i} \square_{T} y_{j}=x_{i} \square_{T}(T-\lambda)^{*} y_{j}=x_{i} \square_{T_{\mid \mathcal{M}}}\left(T_{\mid \mathcal{M}}-\lambda\right)^{*} y_{j},
$$

for all $i, j \in \mathbb{N}$ and finally (via c) of that proposition) that

$$
\left(T_{\mid \mathcal{M}}-\lambda\right)^{*} y_{j}=0
$$

for all $j$.

The subspace $\mathcal{J}:=\operatorname{ker}\left(T_{\mid \mathcal{M}}-\lambda\right)^{*}=\mathcal{M} \ominus \mathcal{N}$ with $\mathcal{N}=((T-\lambda) \mathcal{M})^{-}$ is semi-invariant for $T$, infinite dimensional (it contains the $y_{j}^{\prime} s$ ) and $T_{\mathfrak{J}}(=$ $\left.\left(\left(\left(T_{\mid \mathcal{M}}\right)^{*}\right)_{\mid \mathcal{f}}\right)^{*}\right)=\lambda I_{\mathfrak{f}}$ : it does the required job (note that so would do the a priori smaller subspace $\left.\vee_{j} y_{j}\right)$.

As to the last assertion, observe that $\mathcal{J} \subset \operatorname{ker}\left(T_{\mid \mathcal{M}}-\lambda\right)^{*} \cap \operatorname{ker}\left(T_{\mathcal{F}}-\lambda\right)$ where $\mathcal{F}:=\mathcal{N}^{\perp}$ is invariant for $T^{*}$; (this is easily seen from the $3 \times 3$ triangular block matrix decomposition of $T$ relative to $\mathcal{H}=\mathcal{N} \oplus \mathcal{J} \oplus \mathcal{G}(=\mathcal{M} \oplus \mathcal{G}=\mathcal{N} \oplus \mathcal{F})$ with $\mathcal{G}=\mathcal{M}^{\perp}$

$$
T=\left(\begin{array}{ccc}
T_{\mid \mathcal{N}} & * & * \\
0 & \lambda & * \\
0 & 0 & T_{\mathcal{G}}
\end{array}\right)
$$


Now it is a matter of combining Lemma 3.1. with a systematic use of Prop. 2.2. Let thus $\left(e_{n}\right)_{n}$ a sequence of unit vectors in $\mathcal{J}$ converging weakly to 0 . Since $\left(T_{\mathcal{F}}-\lambda\right) e_{n}=0$ and $\left(e_{n}, e_{n}\right)=1$, we have $e_{n} \square_{T_{\mathcal{F}}} e_{n}=E_{\lambda}$; thus (via Prop. 2.2. c)) $e_{n} \square_{T_{\mathcal{F}}} e_{n}=E_{\lambda}$.

For $w \in \mathcal{H}$, we have (via Prop. 2.2. b)) $w \square_{T} e_{n}=P_{\mathcal{F}} w \square_{T_{\mathcal{F}}} e_{n}$ and this term tends to 0 by Lemma 3.1. applied to $T_{\mathcal{F}}$.

Similarly, $e_{n} \square_{T} w=e_{n} \square_{T_{\mid \mathcal{M}}} P_{\mathcal{M}} w \rightarrow 0$ (via Prop. 2.2. b) for the equality and by Lemma 3.1. applied to $T_{\mid \mathcal{M}}$ for the limit).

Thus we do have $E_{\lambda} \in \mathfrak{X}_{0}(T)$ via the sequences $\left(x_{n}\right)_{n},\left(x_{n}\right)_{n}$.

This being true for any $\lambda$ in $\mathbb{D}$ we obtain (via the absolute convexity and closedness of $\mathfrak{X}_{0}(T)$ ) the desired equality: $\mathfrak{X}_{0}(T)=Q_{1}$.

We can now state and prove our main result.

THEOREM 3.4. Let $T$ be an absolutely continuous $\rho$-contraction. The following assertions are equivalent:

a) The set $\mathfrak{X}_{0}(T)$ contains a ball centered at the origin.

b) The associated functional calculus $\Phi_{T}$ has Property $\left(\mathbb{A}_{\aleph_{0}}\right)$.

c) For each $\lambda \in \mathbb{D}$ there exists a semi-invariant subspace $\mathcal{J}$ for $T$ such that $T_{\mathfrak{\jmath}}=\lambda I_{\mathfrak{\jmath}}$.

d) There exists a semi-invariant subspace $\mathcal{L}$ for $T$ such that $\sigma_{e}\left(T_{\mathcal{L}}\right) \cap \mathbb{D}$ is dominating in $\mathbb{D}$.

e) The approximation set $\mathfrak{X}_{0}(T)$ is maximal, i.e. coincides with the closed unit ball of $Q$.

Proof. We first point out the implications which hold for any absolutely continuous polynomially bounded operator: $e) \Rightarrow a) \Rightarrow b) \Rightarrow c$ ) and $b) \Rightarrow d$ ).

Among these, the first one is obvious, the second one $(a) \Rightarrow b)$ ) is the content of Prop. 2.5., the 3rd one is the first part of Prop. 3.3. and the last one can be proved along the same lines (cf. [3], Prop. 5.4.), whose proof given there for contractions works as well for polynomially bounded operators).

Thus to finish the proof it suffices to prove the implications $d) \Rightarrow b$ ) and $c) \Rightarrow e$ ). The latter follows from the second part of Prop. 3.3. while for $d) \Rightarrow b$ ), under the dominatingness of $\sigma_{e}\left(T_{\mathcal{L}}\right) \cap \mathbb{D}$ we obtain (via Theorem 3.2.) property $\left(\mathbb{A}_{\aleph_{0}}\right.$ ) for $\Phi_{T_{\mathfrak{d}}}$ and consequently (via c) of Prop. 2.6.) also for $\Phi_{T}$. 


\section{References}

[1] H. Bercovici, Factorization theorems and the structure of operators on Hilbert space, Ann. of Math., 128, (1988), 399-413

[2] S. Brown Chevreau, On the structure of contraction operators, II, J. Funct. Anal., 76, (1988), 30-55

[3] H. Bercovici Foias, Dual algebras with applications to invariant subspaces and dilation theory, CBMS Regional Conference Series in Mathematics, No 56, (1985)

[4] S. Brown Chevreau, Toute contraction á calcul fonctionnel isométrique est réflexive, C. R. Acad. Sc. Paris, t. 307, Serie I, (1988), 185-188

[5] I. Chalendar, Techniques d'algèbres duales et sous-espaces invariants, Mémoire de $D E A,(1994)$

[6] I. Chalendar Partington, Modern Approaches to the Invariant-subspace Problem, (2011)

[7] B. Chevreau, Sur les contractions á calcul fonctionnel isométrique, II, J. Operator Theory, 20, (1988)

[8] J. Eschmeier, Invariant subspaces, Introduction to Banach algebras, Operators, and Harmonic Analysis, Ed. by P. Aiena G. Dales J. Eschmeier, London Math. Soc. Student Texts (57), 2003

[9] C. Foiaş B. Sz.-Nagy, Similitude des operateurs de classe $\mathcal{C}_{\rho}$ á des contractions, C. R. Acad. Sc. Paris, Série A, t.264, (1967), 1063-1065

[10] C. Foiaş B. Sz.-Nagy, Harmonic Analysis of Operators on Hilbert Space, Akademiai Kiado, Budapest and North-Holland, Amsterdam-London, 1970

[11] O. Rejasse, Factorization and reflexivity results for polynomially bounded operators, J. Operator Theory, 60, (2008), 219-238

\section{Bernard Chevreau}

Department of Mathematics

Université de Bordeaux

Cours de la libération, 33405

Talence Cédex

FRANCE

E-mail: chevreau@math.u-bordeaux1.fr

Aurelian Crăciunescu

Department of Mathematics

Universitatea de Vest Timişoara

Bul. V. Pârvan nr. 4

300232 Timişoara

ROMANIA 
E-mail: craciunescu@math.uvt.ro

Received: 10.01 .2015

Accepted: 28.02.2015 